\title{
Differences In Gastrointestinal Safety Profiles Among Novel Oral Anticoagulants: Evidence From A Network Meta-Analysis
}

This article was published in the following Dove Press journal: Clinical Epidemiology

\author{
Wen-Qin Guo' \\ Xie-Hui Chen' \\ Xiao-Yuan Tian' \\ Lang $\mathrm{Li}^{2}$
}

'Department of Cardiology, Fuwai Hospital Chinese Academy of Medical Sciences, Shenzhen, People's Republic of China; ${ }^{2}$ Department of Cardiology, The First Affiliated Hospital of Guangxi Medical University, Nanning, People's Republic of China
Correspondence: Lang $\mathrm{Li}$

Department of Cardiology, The First Affiliated Hospital of Guangxi Medical

University, 6 Shuangyong Road, Nanning

53002I, People's Republic of China

Tel +86-77|-533II

Fax +86-77I-535980I

Email gmxu2015@I63.com
Background: There is no consensus at present regarding the differences in the risk of GI bleeding across various NOAC regimens. Therefore, we performed a network meta-analysis to compare the risk of gastrointestinal bleeding after different NOAC regimens.

Methods: PubMed, Cochrane, Web of Science, Clinicaltrial.gov and Clinicaltrialresults.org were searched for randomized controlled trials (RCTs) assessing gastrointestinal bleeding of all NOAC regimens from inception to January 2018. The primary endpoint was major gastrointestinal (MGI) bleeding. The meta-regression was performed to access the association between the MGI bleeding events and mortality. The network meta-analysis was carried out with the Bayesian random-effect model.

Results: A total of 25 RCTs, including 139,392 patients, were identified. Meta-regression analysis showed that MGI bleeding was correlated with fatal bleeding events (odds ratios [OR], 1.76; 95\% confidence interval [CI], 1.13-2.77], $\mathrm{P}=0.015$ ). The network meta-analysis results showed that compared to the conventional regimens, rivaroxaban was associated with increased risk of MGI bleeding (OR, 1.37; 95\% credible interval [CrI], 1.00-1.85), but not the apixaban (OR, 0.77; 95\% CrI, 0.53-1.07]), edoxaban (OR, 0.86; 95\%CrI, 0.52-1.18) and dabigatran etexilate (OR, 1.22; 95\% CrI, 0.82-1.69). Compared to rivaroxaban, apixaban (OR, 0.56; 95\% CrI, 0.35-0.88) and edoxaban (OR, 0.62; 95\% CrI, 0.35-0.96) showed a significantly lower risk of MGI bleeding. Apixaban had the highest probability of being the safest option with regard to the risk of MGI bleeding $(89.1 \%)$, followed by edoxaban $(77.4 \%)$, conventional therapy $(51.4 \%)$, dabigatran etexilate $(23.8 \%)$ and rivaroxaban $(8.3 \%)$.

Conclusion: The risk of GI bleeding significantly varies among different NOAC regimens, and evidence shows that apixaban and edoxaban had the most favorable MGI bleeding safety profile, while rivaroxaban and dabigatran etexilate were the least safe.

Keywords: new oral anticoagulant, gastrointestinal bleeding, network meta-analysis

\section{Background}

New oral anticoagulants (NOACs) are effective in the prevention and treatment of thrombosis, and thus have become the main therapeutic drugs for the prevention of venous thromboembolism (VTE) and ischemic stroke prevention in atrial fibrillation (AF). ${ }^{1}$ The European Society of Cardiology (ESC) Guidelines for the Management of AF include a class IA recommendation, i.e. they recommend NOAC as the preferred drug for AF prevention instead of warfarin. ${ }^{2}$ The 10th edition of the American College of Chest Physicians (ACCP) Guidelines for VTE 
Disease also recommended NOAC over vitamin $\mathrm{K}$ antagonist (VKA) therapy for treating VTE (Grade 2B). ${ }^{3}$ However, none of the current guidelines have specific recommendations regarding the preferred NOAC regimen.

Recent studies have shown that the risk of gastrointestinal (GI) bleeding depends on the NOAC regimen. The Rivaroxaban Once Daily Oral Direct Factor Xa Inhibition Compared with Vitamin K Antagonism for Prevention of Stroke and Embolism Trial in Atrial Fibrillation (ROCKET AF) trial revealed that rivaroxaban significantly increased the risk of GI bleeding compared with warfarin. ${ }^{4}$ On the contrary, there is also evidence that the risk of GI bleeding in patients taking oral apixaban is lower than that in the warfarin group. ${ }^{5}$ However, no randomized-controlled trial (RCT) has directly compared the risk of GI bleeding following different NOAC regimens. Therefore, there is no consensus at present regarding the differences in the risk of GI bleeding across various NOAC regimens.

To clarify this issue, we used network meta-analysis, an emerging methodology which uses a common comparison group to make indirect comparisons of all interventions. ${ }^{6}$ All relevant clinical studies were reviewed, and the risk of GI bleeding in all NOAC regimens was analyzed using Bayesian network meta-analysis in order to provide guidance for clinical decision-making.

\section{Methods}

This study was performed in accordance with the Preferred Reporting Items for Systematic Reviews and Meta-Analysis statement. $^{7}$

\section{Data Sources And Searches}

The PubMed, the Cochrane library, Web of Science, Clinicaltrial.gov, Clinicaltrialresults.org and reference lists of relevant papers were searched from inception to January 2018. The key words were as follows: "atrial fibrillation," "deep vein thrombosis," "pulmonary embolism," "venous thromboembolism," "novel oral anticoagulants" (including "edoxaban," "apixaban," "rivaroxaban," "dabigatran etexilate").

\section{Study Selection}

We included studies if: 1) the recruited population should be patients with atrial fibrillation, deep vein thrombosis or pulmonary embolism; 2) the intervention should be the NOACs, including edoxaban, apixaban, rivaroxaban, and dabigatran etexilate; 3) the comparator should be the NOACs or conventional therapy (including warfarin, heparin and placebo); 4) reported any occurrence of major gastrointestinal bleeding; 5) were randomized controlled trials (RCTs); 6) were published in English. We excluded studies with zero events in both groups because these comparisons provide no information on the treatment effect. Additionally, we excluded studies assessing NOAC in patients with acute coronary syndrome. We also excluded studies assessing NOAC in AF patients treated with ablation catheter or percutaneous coronary intervention. Because in these studies, other drugs (e.g. antiplatelet, VKA, etc.) known to cause MGI bleeding were used. If different results were noted in the same trial, we extracted the most recent data for analysis.

\section{Data Extraction And Assessment Of Study Quality}

Two independent investigators (W.Q.G., X.H.C.) reviewed the full text of eligible studies and extracted the information. To reach a consensus, a divergence of views was resolved by joint review of the literature. Conflicts will be resolved by the third investigator (L.L.). The Cochrane risk-of-bias tool was used to assess the study quality for $\mathrm{RCT}^{8}$

\section{Outcomes Measures}

The primary outcome was an occurrence of major gastrointestinal bleeding, which was defined according to the individual trials.

\section{Data Synthesis And Statistical Analysis}

In the pairwise meta-analysis, we assessed all clinical outcomes by calculating odds ratios (ORs) and corresponding 95\% confidence intervals (CIs). To account for unexplained heterogeneity, we performed the meta-analysis using a random-effects model (DerSimonian-Laird method). ${ }^{9}$ The Cochrane $\mathrm{Q}$ test and the inconsistency index $\left(\mathrm{I}^{2}\right)$ test were used to assess statistical heterogeneity. ${ }^{10} \mathrm{I}^{2}$ values less than $25 \%$ were indicative of low heterogeneity, while values between $25 \%$ and $50 \%$ were indicative of moderate heterogeneity, and values greater than $50 \%$ were indicative of high heterogeneity. The funnel plot method and Egger's regression asymmetry test were used to determine publication bias if the number of studies was more than $10 .{ }^{11}$ We hypothesized that in the contemporary era of NOAC, major bleeding had an impact on mortality. To explore this hypothesis, we performed a meta-regression of the effects sizes of MGI bleeding on mortality. ${ }^{12}$ The ORs for treatment effects in individual trials were log-transformed before being used as 
independent variables in linear meta-regression analyses. The pairwise meta-analysis and meta-regression were performed using STATA software, version 12.0.

The network meta-analysis was carried out modelling the binary outcome with the Bayesian framework using the Markov chain Monte Carlo methods. ${ }^{13}$ The analysis was based on informative priors for effect sizes. All outcomes were expressed as ORs and its corresponding 95\% credibility intervals (CrIs). Three chains were fit, yielding 300,000 iterations $(100,000$ per chain) generating the posterior distributions of model parameters. Convergence was checked by the Brooks-Gelman-Rubin diagnostic. ${ }^{14}$ We selected the random effect model because of the unexplained heterogeneity. ${ }^{13,15}$ Model fit was assessed with deviance information criterion. ${ }^{13} \mathrm{I}^{2}$ statistic was used to investigate the possibilities of statistical heterogeneity. ${ }^{16}$ Consistency between direct and indirect estimates was appraised comparing consistency and inconsistency models. ${ }^{17}$ In addition, we evaluated rank probabilities by using surface under cumulative ranking (SUCRA) to determine the probability that each treatment is the best in terms of MGI bleeding outcome. ${ }^{18}$ To explore the association between risk of MGI bleeding and the length of study follow-up, we performed meta-regression where treatment effects relative to conventional therapy were allowed to depend on the study follow-up through a single interaction term. ${ }^{16}$ Additionally, we conducted subgroup analysis of the data by calculating the interaction term $\beta .{ }^{16}$ We considered the age group (mean age $<65$ versus $\geq 65$ ); the proportion of male $(<50 \%$ versus $\geq 50 \%)$; the indication for taking NOAC (VTE, AF and CAD/PAD) as the variables. We also plotted a comparison-adjusted funnel plot for the network meta-analysis, to detect the presence of any dominant publication bias in network meta-analysis. ${ }^{19}$ Bayesian frame network meta-analysis was conducted by means of gemtc, rjags in $\mathrm{R}$ software, version 3.2.0 ( $\mathrm{R}$ Foundation for Statistical Computing) and NetMetaXL. ${ }^{20,21}$

\section{Results}

\section{Study Selection And Characteristics}

A flow diagram of the literature search and study selection process is shown in Figure 1. We found 1686 studies from a search of PubMed, the Cochrane library, Web of Science, Clinicaltrial.gov, Clinicaltrialresults.org and reference lists of relevant papers. The total number of article was 1061 after removing the duplicated records. Twenty-eight studies were included in quantitative synthesis after the full-text evaluation.
There were four studies with zero MGI bleeding events in both groups. Finally, 24 studies with 25 RCTs involving 139,392 patients met the inclusion criteria. ${ }^{5,22-44}$ One study was the report of two trials (RE-MEDY and RE-SONATE trial). ${ }^{40}$ The network plot of the comparisons is given in Figure 2. The following options were tested in the network: edoxaban, apixaban, rivaroxaban, dabigatran etexilate, and conventional therapy. The conventional therapy included warfarin, heparin, and placebo. Among these 25 RCTs, 7 compared apixaban with conventional therapy, ${ }^{5,22-27} 5$ compared dabigatran etexilate with conventional therapy, ${ }^{34,39,40,43} 6$ compared edoxaban with conventional therapy ${ }^{28,31-33,42,44}$ and 7 trial compared rivaroxaban with conventional therapy. ${ }^{29,30,35,36,38,41}$ The characteristics of the studies included in the analysis and their populations are listed in Table 1. Seven trials included the patients with $\mathrm{AF}^{26-28,32,38,39,41} 1$ trial included patients with $\mathrm{CAD} / \mathrm{PAD},{ }^{30}$ and 17 trials included patients with VTE. ${ }^{5,22-25,29,31,33-37,40,42-44}$ The mean age of patients ranged from 54 to 73 years. The proportion of male ranged from 14\% to $81 \%$. The follow-up time ranged from 12 days to 2.8 years. The definition of the Major bleeding in all the studies was according to the criterial of International Society on Thrombosis and Haemostasis for bleeding in nonsurgical patients ${ }^{45}$ (shown in Additional file: Table S1). An assessment of the risk of bias of the included studies is presented in Additional file: Figure S1. Overall, the included studies were found to have a low risk of bias.

\section{The Results From Pair-Wise Meta- Analysis And Meta-Regression}

The results of pairwise meta-analysis are displayed in Figure 3. The rivaroxaban was associated with an increased risk of MGI bleeding compared with the conventional therapy (OR 1.35, 95\% CI: 1.03-1.77, $\mathrm{P}=0.030$ ). The apixaban ( $\mathrm{OR}=0.72, \quad 95 \% \quad$ CI: $0.46-1.12, \quad \mathrm{P}=0.144)$, edoxaban (OR=0.89, 95\% CI: $0.68-1.15, \mathrm{P}=0.360)$ and dabigatran etexilate $(\mathrm{OR}=1.17,95 \%$ CI: $0.80-1.71, \mathrm{P}=0.415)$ had a similar risk of MGI bleeding relative to the conventional therapy. The meta-regression showed a significant association between MGI bleeding and fatal bleeding mortality across individual studies (17 trials; OR: $1.76,95 \% \mathrm{CI}$ : 1.13-2.77, $\mathrm{P}=0.015$ ) (Figure 4).

\section{The Results From Network Meta-Analysis}

The results of network meta-analysis are shown in Table 2. Compared to conventional therapy, rivaroxaban was associated with increased risk of MGI bleeding (OR, 1.37; 


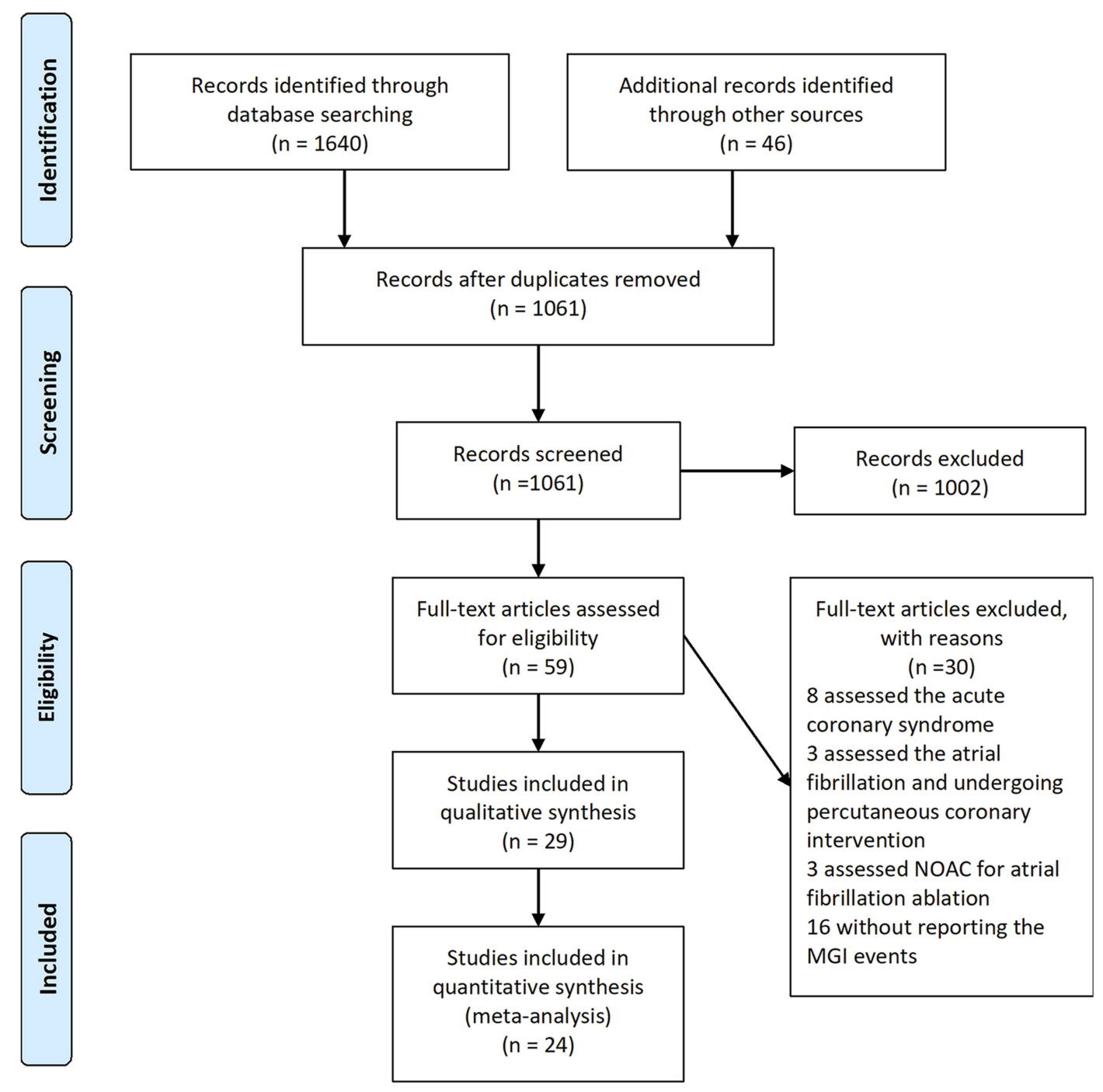

Figure I PRISMA flow diagram of study selection.

95\% CrI, 1.00-1.85). For apixaban (OR, 0.77; 95\% CrI, 0.53-1.07), edoxaban (OR, 0.86; 95\% CrI, 0.52-1.18) and dabigatran etexilate (OR,1.22; 95\% CrI, 0.82-1.69), evidence was lacking for an increased risk of MGI bleeding compared with conventional therapy. Relative to rivaroxaban, the pooled results favored apixaban with an OR of 0.56 (95\% CrI, 0.35-0.88), edoxaban with an OR of 0.62 (95\% CrI, 0.35-0.96). No difference of MGI bleeding risk was observed between the rivaroxaban and dabigatran etexilate (OR, 1.34; 95\% CrI, 0.71-1.85). Compared with dabigatran etexilate, apixaban (OR, 0.64; 95\% CrI, 0.391.04) had a trend to reduce the MGI bleeding risk, although the difference was not significant. The risk of MGI bleeding was similar between the edoxaban and dabigatran etexilate (OR, 0.70; 95\% CrI, 0.39-1.14).
Also, the apixaban had a similar risk of MGI bleeding relative to the edoxaban (OR, 0.91; 95\% CrI, 0.56-1.58).

The probability ranking of each treatment is shown in Figure 5. Apixaban had the highest probability to be the safest option with regard to the risk of MGI bleeding (89.1\%), followed by edoxaban (77.4\%), conventional therapy (51.4\%), dabigatran etexilate $(23.8 \%)$ and rivaroxaban $(8.3 \%)$.

The common heterogeneity SD was $0.15(95 \% \mathrm{CrI}$ $0.04-0.44)$. The global heterogeneity parameter $\mathrm{I}^{2}$ values were $40.3 \%$ (Additional file: Figure S2). The inconsistency cannot exist because the network is star-shaped. Bayesian meta-regressions showed no significant effect for age, sex of the patients, indications for NOAC and study follow-up (Additional file: Table S2). The 


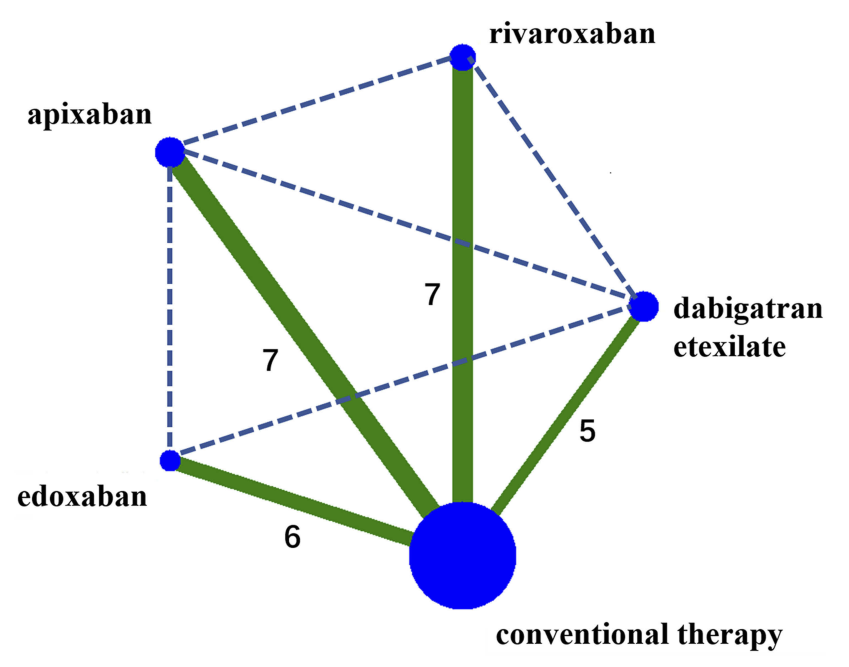

Figure 2 The network plot for the Bayesian meta-analysis.

Notes: Solid lines represent direct evidence, and dashed lines represent the indirect evidence. The size of the nodes corresponds to the number of patients receiving the regimen, and the width of the lines corresponds to the number of studies comparing the connected treatments.

comparison-adjusted funnel plots suggest that no publication bias was present (Additional file: Figure S3).

\section{Discussion}

Our network meta-analysis evaluated a large number of studies that compared the risk of GI bleeding following four different NOAC regimens. Our main findings were that 1) the MGI bleeding events were correlated with fatal bleeding events, 2) the extent of MGI bleeding differed among the individual NOAC regimens, with edoxaban and apixaban associated with a lower risk compared to rivaroxaban, and 3) apixaban was the safest option with regards to MGI bleeding, followed by edoxaban, dabigatran etexilate and rivaroxaban.

\section{Current Understanding Of The Risk Of Post-NOAC GI Bleeding}

Several studies have demonstrated that NOACs have better antithrombotic effect than warfarin and can significantly reduce mortality rate. ${ }^{26,46-48}$ Therefore, NOAC has steadily replaced warfarin as the preferred drug for thrombosis prevention and treatment. ${ }^{2}$ Although the overall risk of bleeding is lower following NOAC than warfarin, ${ }^{49}$ the risk of GI bleeding is still higher with the former, ${ }^{50}$ suggesting that active prevention and treatment of post-NOAC GI bleeding are vital during clinical operations. The secondary analysis of the ROCKET-AF trial compared the risk of GI bleeding between patients treated with oral rivaroxaban and those treated with warfarin. ${ }^{4}$ Of the 14,236 AF patients enrolled in this trial, 684 had GI bleeding during follow-up, and rivaroxaban was associated with higher risk of GI bleeding than warfarin. Conversely, the Apixaban after the Initial Management of Pulmonary Embolism and Deep Vein Thrombosis with First-Line Therapy-Extended Treatment (AMPLIFY-EXT) trial showed that the risk of GI bleeding was significantly lower after oral apixaban than after warfarin. $^{25}$ Since there is currently no head-to-head RCT that compares all NOAC regimens, there is no definite consensus on whether the risk of post-NOAC GI bleeding is different between the specific regimens.

Nevertheless, observational studies have yielded significant evidence. A large cohort study conducted by Abraham et al involving 31,574 patients was carried out to compare the risk of GI bleeding following dabigatran, rivaroxaban, and apixaban regimens. ${ }^{51}$ Compared to dabigatran or rivaroxaban, apixaban significantly reduced the risk of GI bleeding. Furthermore, the risk of GI bleeding was higher after rivaroxaban than after dabigatran, but not statistically significant. This finding is consistent with the results from our network meta-analysis. In our study, we found that the MGI bleeding risk was significantly higher with rivaroxaban (OR, 1.79; 95\% CrI, 1.14-2.86) and non-significantly higher for dabigatran when relative to the apixaban (OR, 1.56; 95\% CrI, 0.962.56). However, since large-scale clinical trials are complicated and cumbersome, Abraham et al were unable to include edoxaban into the comparison of intervention measures, and therefore the risk of post-edoxaban GI bleeding could not be evaluated. In our analysis, edoxaban and apixaban had lower risk of MGI bleeding than rivaroxaban, although no difference was observed between edoxaban and apixaban $(\mathrm{OR}=0.91$, and the SUCRA values were $86.1 \%$ and $76.9 \%$, respectively). Therefore, apixaban and edoxaban had the most favorable MGI bleeding safety profile. On the contrary, the MGI bleeding was similar between dabigatran and rivaroxaban, with comparable SUCRA values $(28.0 \%$ and $10.9 \%$, respectively), indicating that these two drugs had the least favorable MGI bleeding safety profile.

In addition, although several observational studies have compared the risk of GI bleeding after different NOAC regimens, ${ }^{52-56}$ they have several limitations like inconsistent definition of GI bleeding, presence of selective bias due to the nature of study (observational), and lack of comparative study of all NOAC regimens. Therefore, it is difficult to compare the results of these studies with those from our network meta-analysis. 
Table I The Characteristic Of The Included Studies

\begin{tabular}{|c|c|c|c|c|c|c|}
\hline Study & Intervention & $\begin{array}{l}\text { Regimen In The } \\
\text { CT Group }\end{array}$ & $\begin{array}{l}\text { Follow-Up } \\
\text { Time }\end{array}$ & $\begin{array}{l}\text { Median } \\
\text { Age (y) }\end{array}$ & Male (\%) & $\begin{array}{l}\text { Indication For } \\
\text { Taking NOCA }\end{array}$ \\
\hline ADVANCE $2009^{24}$ & Apixaban & Enoxaparin & 12 days & 65.8 & 37.9 & VTE \\
\hline ADVANCE-2 $2010^{23}$ & Apixaban & Enoxaparin & 12 days & 67 & 27.5 & VTE \\
\hline ADVANCE-3 $20102^{22}$ & Apixaban & Enoxaparin & 35 days & 60.8 & 46.7 & VTE \\
\hline AMPLIFY $20 I^{34}$ & Apixaban & Warfarin & 6 months & 57 & 58.7 & VTE \\
\hline AMPLIFY EXT $2013^{25}$ & Apixaban & Placebo & 12 months & 56.7 & 57.4 & VTE \\
\hline ARISTOTLE $20 \mathrm{II}^{26}$ & Apixaban & Warfarin & 1.8 years & 70 & 64.7 & $\mathrm{AF}$ \\
\hline AVERROES $20 \mathrm{II}^{27}$ & Apixaban & Aspirin & I.I years & 70 & 58.5 & $\mathrm{AF}$ \\
\hline RE-COVER $2009^{34}$ & $\begin{array}{l}\text { Dabigatran } \\
\text { etexilate }\end{array}$ & Warfarin & 163 days & 54.7 & 58.4 & VTE \\
\hline RE-COVER II $2014^{43}$ & $\begin{array}{l}\text { Dabigatran } \\
\text { etexilate }\end{array}$ & Warfarin & 164 days & 54.9 & 60.6 & VTE \\
\hline RE-MEDY $2013^{40}$ & $\begin{array}{l}\text { Dabigatran } \\
\text { etexilate }\end{array}$ & Warfarin & 473 days & 54.7 & 60.9 & VTE \\
\hline RE-SONATE $2013^{40}$ & $\begin{array}{l}\text { Dabigatran } \\
\text { etexilate }\end{array}$ & Placebo & 164 days & 55.8 & 55.5 & VTE \\
\hline RELY $2009^{39}$ & $\begin{array}{l}\text { Dabigatran } \\
\text { etexilate }\end{array}$ & Warfarin & 2 years & 71.5 & 63.6 & $\mathrm{AF}$ \\
\hline Chung, et al $201 \mathrm{I}^{32}$ & Edoxaban & Warfarin & 3 months & 65.1 & 65.4 & $\mathrm{AF}$ \\
\hline ENGAGE-AF-TIMI ${ }^{47} 2013^{28}$ & Edoxaban & Warfarin & 2.8years & 72 & 61.9 & $\mathrm{AF}$ \\
\hline Fuji, et al $2014^{31}$ & Edoxaban & Enoxaparin & 12 days & 76.1 & 20.5 & VTE \\
\hline Hakusai-VTE $2013^{33}$ & Edoxaban & Warfarin & 250 days & 55.8 & 57.2 & VTE \\
\hline STARS E-3 $2014^{42}$ & Edoxaban & Enoxaparin & 13 days & 72.3 & 20.2 & VTE \\
\hline STARS J-V $2015^{44}$ & Edoxaban & Enoxaparin & 13 days & 62.8 & |4. 1 & VTE \\
\hline COMPASS $2017^{30}$ & Rivaroxaban & Placebo & 23 months & 68.2 & 22 & CAD/PAD \\
\hline EINSTEIN $2010^{29}$ & Rivaroxaban & Placebo & 264 days & 56.1 & 56.8 & VTE \\
\hline J-ROCKET AF $2012^{38}$ & Rivaroxaban & Warfarin & 30 months & 71.1 & 80.6 & $\mathrm{AF}$ \\
\hline RECORDI $2008^{36}$ & Rivaroxaban & Enoxaparin & 35 days & 63.2 & 44.5 & VTE \\
\hline RECORD2 $2008^{35}$ & Rivaroxaban & Enoxaparin & 35 days & 61.5 & 46.4 & VTE \\
\hline RECORD4 $2009^{37}$ & Rivaroxaban & Enoxaparin & 12 days & 64.6 & 34.9 & VTE \\
\hline ROCKET AF $201 I^{4 l}$ & Rivaroxaban & Warfarin & 590 days & 73 & 60.3 & $\mathrm{AF}$ \\
\hline
\end{tabular}

Abbreviations: VTE, venous thromboembolism; AF, atrial fibrillation; CAD, coronary artery disease; PAD, peripheral artery disease; CT, conventional therapy.

Several meta-analyses have in fact assessed the risk of GI bleeding following NOAC. Bai et al found that the risk of GI bleeding was higher after rivaroxaban compared to warfarin. ${ }^{57}$ Another meta-analysis including 28 real-word studies showed that rivaroxaban increased the risk of GI bleeding compared with warfarin, while apixaban decreased the risk. ${ }^{46}$ These results are consistent with our findings. It is important to note that these studies used traditional meta-analysis method and failed to compare the MGI bleeding among all NOAC regimens. Burr et al used network meta-analysis to assess the risk of bleeding following NOAC in 31 RCTs using GI bleeding event as the primary endpoint. ${ }^{58}$ The authors found that compared to warfarin and low-molecular weight heparin, NOAC did not increase the risk of GI bleeding. Similar to the aforementioned studies, the study of Burr et al did not further examine the difference in the risk of GI bleeding between various NOAC treatments. Our network meta-analysis includes all relevant studies on the risk of GI bleeding following NOACs and is currently the first large network metaanalysis including 25 RCTs and 139,392 patients. In addition, we assessed and compared the risk of MGI bleeding among the four commonly used clinical NOAC regimens. The results suggest that the risk of MGI bleeding was different between the individual NOACs. Previous studies reported relevant sex differences in the clinical effectiveness of antithrombotic therapy. ${ }^{59,60}$ However, in our research, the meta-regression showed that the sex was not related to the MGI bleeding of NOACs.

\section{Clinical Implications Of The Study}

This study evaluated the relationship between post-NOAC GI bleeding and death, as well as the difference in the risk 


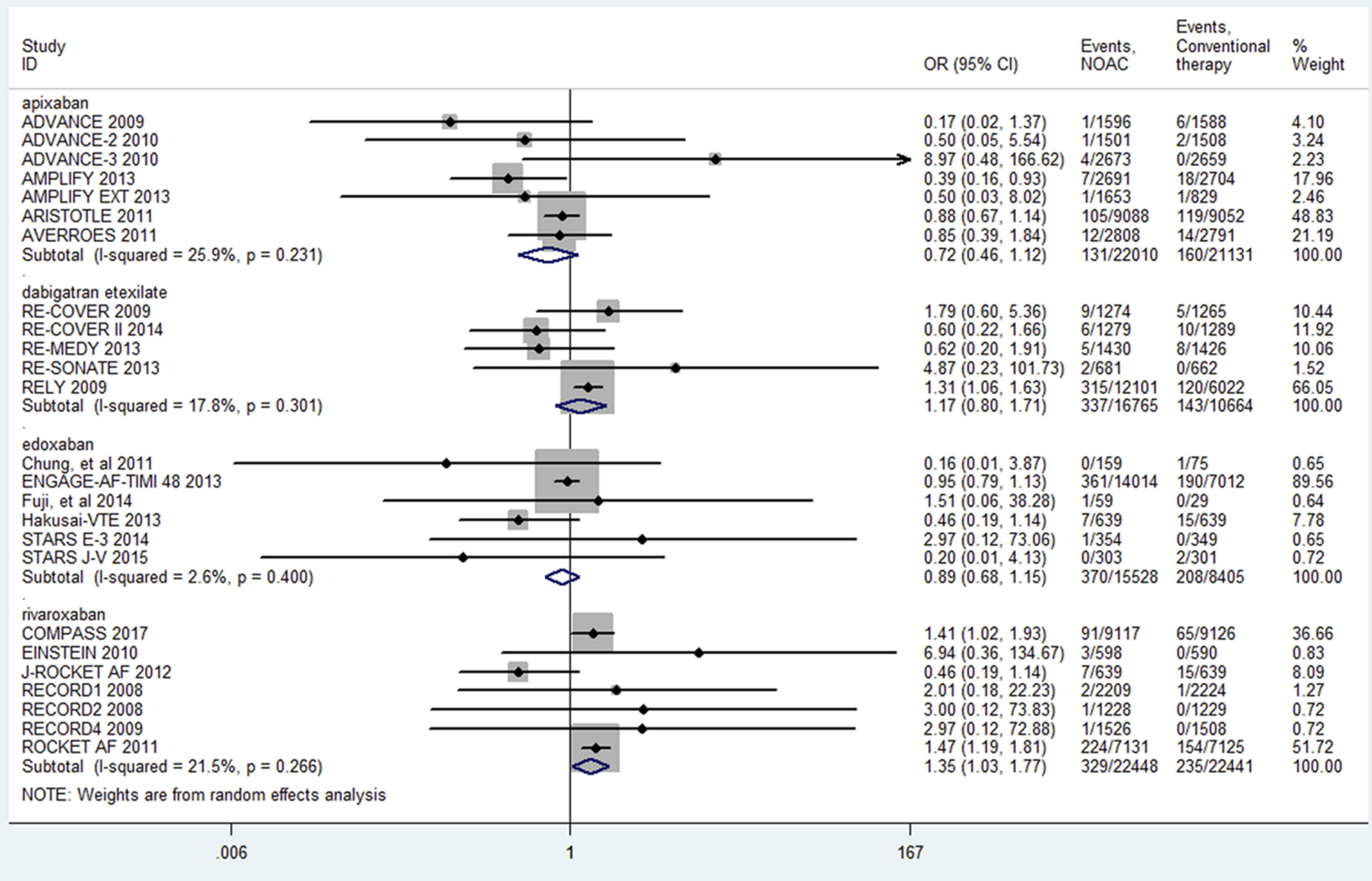

Figure 3 Results of the pairwise meta-analysis.

Notes: Forest plots illustrating the results of a meta-analysis. The calculated summary effect demonstrates the superiority of various NOACs compared with conventional therapy in reducing the risk of major gastrointestinal bleeding. Horizontal lines represent the $95 \% \mathrm{Cl}$ of the effect size; solid diamonds indicate the mean effect size in single studies; hollow diamond shapes depict the summary effect size (diamond center) and the relative $95 \% \mathrm{Cl}$ (lateral edges); the black vertical lines represent the reference "I" line.

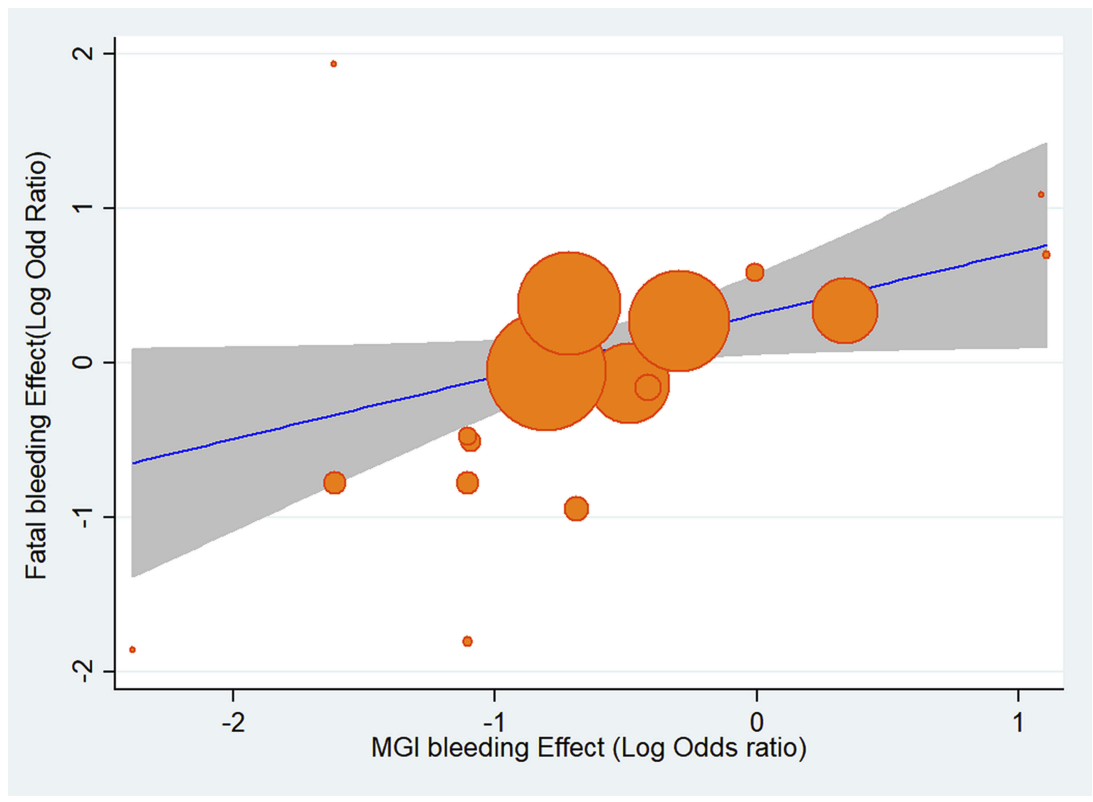

Figure 4 Meta-regression analysis of the effect of MGI bleeding event rate on the effect of fatal bleeding event rate.

Abbreviation: MGI, major gastrointestinal. 
Table 2 Estimated Relative Treatment Effects As Odds Ratios (ORs) And Its Corresponding 95\% Credibility Intervals (Crls)

\begin{tabular}{|l|l|l|l|l|l|}
\hline Treatment & Apixaban & Edoxaban & Rivaroxaban & Dabigatran Etexilate & Conventional Therapy \\
\hline Apixaban & - & $1.09(0.63-1.79)$ & $1.79(1.14-2.86)$ & $1.56(0.96-2.56)$ & $1.30(0.93-1.89)$ \\
Edoxaban & $0.91(0.56-1.58)$ & - & $1.61(1.04-2.86)$ & $1.43(0.88-2.56)$ & $1.16(0.85-1.92)$ \\
Rivaroxaban & $0.56(0.35-0.88)$ & $0.62(0.35-0.96)$ & - & $0.88(0.54-1.40)$ & $0.73(0.54-1.00)$ \\
Dabigatran Etexilate & $0.64(0.39-1.04)$ & $0.70(0.39-1.14)$ & $1.34(0.71-1.85)$ & - & $0.82(0.59-1.22)$ \\
Conventional Therapy & $0.77(0.53-1.07)$ & $0.86(0.52-1.18)$ & $1.37(1.00-1.85)$ & $1.22(0.82-1.69)$ & - \\
\hline
\end{tabular}

Notes: Comparisons should be read from left to right. The estimate is located at the intersection of the column-defining treatment and the row-defining treatment. An OR value higher than I favors the column-defining treatment. An OR value lower than I favors the row-defining treatment.

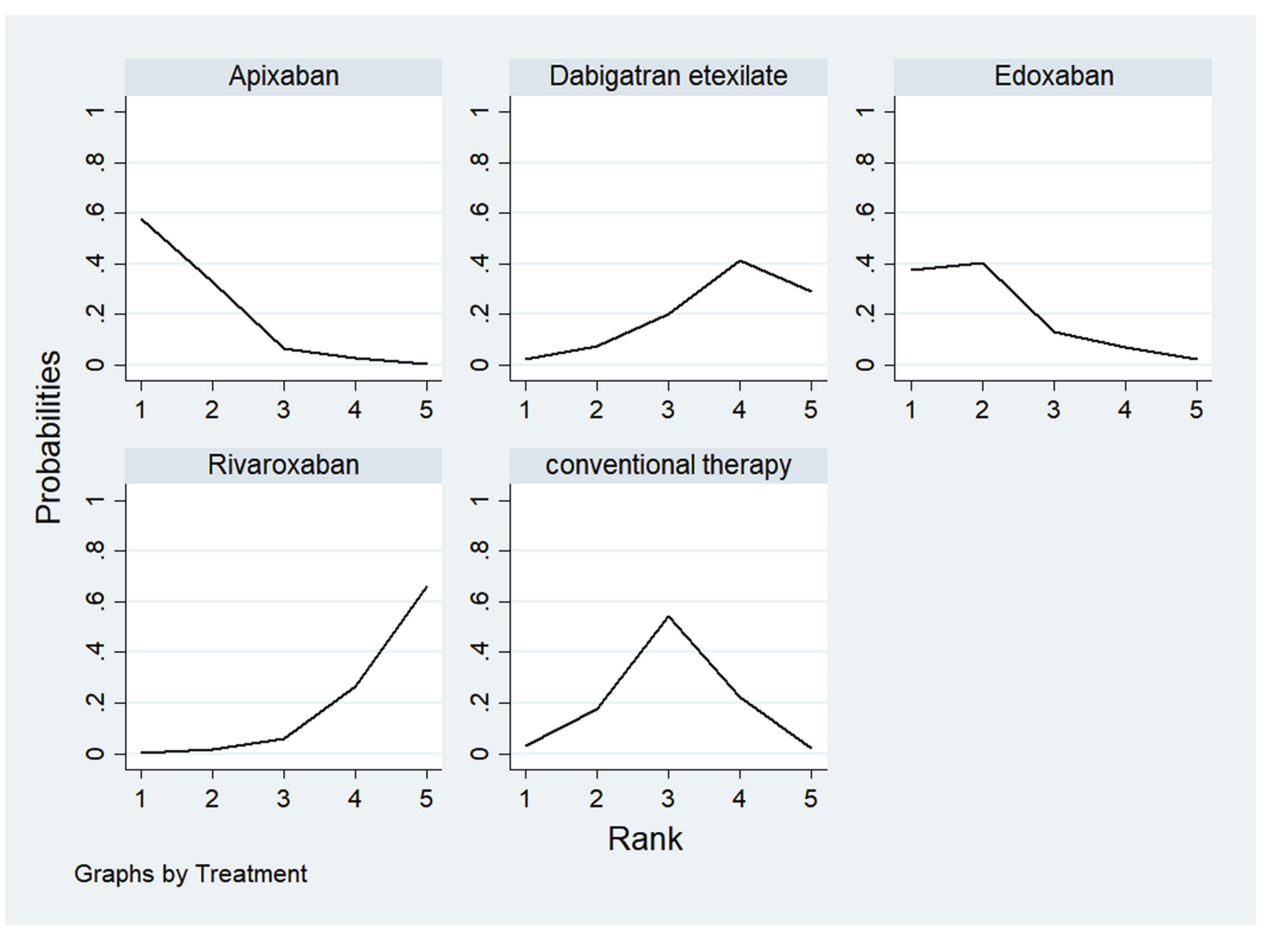

Figure 5 Graph showing probability ranking of each NOAC regimen for the risk of MGI bleeding.

Note: Rank $I$ is best and rank 5 is worst.

of GI bleeding between the four NOAC regimens. According to our findings, GI bleeding was correlated with fatal bleeding following all NOAC treatments with an overall incidence rate of $1.6 \%$, and the risk of GI bleeding differed across NOAC types. Of note, subgroup analysis showed that age, sex of the patients, and indications for NOAC had no effect on the risk of GI bleeding. As the number of NOAC users steadily increases around the world, the occurrence of post-NOAC GI bleeding must be carefully monitored. Additionally, in patients with MGI bleeding associated with the use of a factor $\mathrm{Xa}$ inhibitor, treatment with andexanet significantly reduced anti-factor Xa activity. ${ }^{61}$ However, most countries do not have such drugs, making it challenging to manage NOAC-associated MGI bleeding. Moreover, given that post-NOAC GI bleeding can affect the prognosis of patients, the risk of bleeding in such patients should be carefully evaluated. Since the risk of GI bleeding depends on the exact NOAC regimen, the drug with the lowest risk of bleeding should be selected for clinical use.

\section{Limitations Of The Study}

This study has several limitations. First, the incidence of small GI bleeding events was not analyzed due to lack of sufficient data. Nevertheless, since MGI bleeding events are more serious and often affect clinicians' choice of drugs, it is of greater clinical significance to use MGI bleeding event as the primary endpoint. Second, most studies that were analyzed used other background drugs that might have lead to GI bleeding. However, our study only included RCTs in which 
the confounding factors had already been eliminated during randomization, and likely did not affect our evaluation of the relationship between NOAC and MGI bleeding. Third, given the nature of these RCTs, we could not obtain individual patient data and identify those who are at high risk of MGI bleeding based on demographic and/or clinical characteristics. Fourth, our study did not evaluate the efficacy of the four NOAC regimens. However, there are other network metaanalysis studies that have already confirmed that treatment efficacies of the four regimens were similar. ${ }^{62,63}$ Finally, our study did not carry out a cost-benefit analysis, and therefore we could not assess dosage from an economic perspective.

\section{Conclusion}

The risk of MGI bleeding significantly varies among the NOAC regimens, with apixaban and edoxaban having the most favorable MGI bleeding safety profile, and rivaroxaban and dabigatran being the least safe.

\section{Abbreviations}

NOAC, new oral anticoagulants; RCT, randomized controlled trials; MGI, major gastrointestinal; VTE, venous thromboembolism; AF, atrial fibrillation; VKA, vitamin $\mathrm{K}$ antagonist; CI, confidence interval; CrI, credible interval; OR, odds ratios; SUCRA, surface under cumulative ranking.

\section{Availability of data and materials}

All data generated or analyzed during this study are included in this article and its additional files.

\section{Disclosure}

The authors declare that they have no competing interests.

\section{References}

1. Lane DA, Wood K. Cardiology patient page. Patient guide for taking the non-vitamin $\mathrm{K}$ antagonist oral anticoagulants for atrial fibrillation. Circulation. 2015;131(16):e412-e415. doi:10.1161/CIRCULATIONA HA.114.012808

2. Kirchhof P, Benussi S, Kotecha D, et al. 2016 ESC guidelines for the management of atrial fibrillation developed in collaboration with EACTS. Europace. 2016;18(11):1609-1678. doi:10.1093/europace/ euw295

3. Kearon C, Akl EA, Ornelas J, et al. Antithrombotic therapy for VTE disease: CHEST guideline and expert panel report. Chest. 2016;149 (2):315-352. doi:10.1016/j.chest.2015.11.026

4. Sherwood MW, Nessel CC, Hellkamp AS, et al. Gastrointestinal bleeding in patients with atrial fibrillation treated with rivaroxaban or warfarin: ROCKET AF trial. $J$ Am Coll Cardiol. 2015;66(21):22712281. doi:10.1016/j.jacc.2015.09.024

5. Agnelli G, Buller HR, Cohen A, et al. Oral apixaban for the treatment of acute venous thromboembolism. N Engl J Med. 2013;369(9):799808. doi:10.1056/NEJMoa1302507
6. Glenny AM, Altman DG, Song F, et al. Indirect comparisons of competing interventions. Health Technol Assess (Rockv). 2005;9 (26):1-+.

7. Moher D, Liberati A, Tetzlaff J, Altman DG, Group P. Preferred reporting items for systematic reviews and meta-analyses: the PRISMA statement. Ann Intern Med. 2009;151(4):264-269, W264. doi:10.7326/0003-4819-151-4-200908180-00135

8. Higgins JP, Altman DG, Gotzsche PC, et al. The cochrane collaboration's tool for assessing risk of bias in randomised trials. BMJ. 2011;343:d5928. doi:10.1136/bmj.d5928

9. DerSimonian R, Laird N. Meta-analysis in clinical trials. Control Clin Trials. 1986;7(3):177-188.

10. Higgins JP, Thompson SG, Deeks JJ, Altman DG. Measuring inconsistency in meta-analyses. BMJ. 2003;327(7414):557-560. doi:10. 1136/bmj.327.7414.557

11. Sterne JA, Egger M. Funnel plots for detecting bias in meta-analysis: guidelines on choice of axis. J Clin Epidemiol. 2001;54(10):10461055. doi:10.1016/s0895-4356(01)00377-8

12. Thompson SG, Higgins JPT. How should meta-regression analyses be undertaken and interpreted? Stat Med. 2002;21(11):1559-1573. doi:10.1002/sim.1187

13. Dias S, Sutton AJ, Ades AE, Welton NJ. Evidence synthesis for decision making 2: a generalized linear modeling framework for pairwise and network meta-analysis of randomized controlled trials. Med Decis Making. 2013;33(5):607-617. doi:10.1177/0272989X1 2458724

14. Brooks SP, Gelman A. General methods for monitoring convergence of iterative simulations. J Comput Graph Stat. 1998;7:434-455.

15. Mills EJ, Thorlund K, Ioannidis JP. Demystifying trial networks and network meta-analysis. BMJ. 2013;346:f2914. doi:10.1136/bmj.f1164

16. Dias S, Sutton AJ, Welton NJ, Ades AE. Evidence synthesis for decision making 3: heterogeneity - subgroups, meta-regression, bias, and bias-adjustment. Med Decis Making. 2013;33(5):618-640. doi: $10.1177 / 0272989 X 13485157$

17. Dias S, Welton NJ, Caldwell DM, Ades AE. Checking consistency in mixed treatment comparison meta-analysis. Stat Med. 2010;29(78):932-944. doi:10.1002/sim.3767

18. Salanti G, Ades AE, Ioannidis JP. Graphical methods and numerical summaries for presenting results from multiple-treatment meta-analysis: an overview and tutorial. J Clin Epidemiol. 2011;64(2):163171. doi:10.1016/j.jclinepi.2010.03.016

19. Chaimani A, Higgins JP, Mavridis D, Spyridonos P, Salanti G. Graphical tools for network meta-analysis in STATA. PLoS One. 2013;8(10):e76654. doi:10.1371/journal.pone.0076654

20. van Valkenhoef G, Lu G, de Brock B, Hillege H, Ades AE, Welton NJ. Automating network meta-analysis. Res Synth Methods. 2012;3 (4):285-299. doi:10.1002/jrsm.1054

21. Brown S, Hutton B, Clifford T, et al. A microsoft-excel-based tool for running and critically appraising network meta-analyses - an overview and application of NetMetaXL. Syst Rev. 2014;3:110. doi:10.1186/2046-4053-3-110

22. Lassen MR, Gallus A, Raskob GE, Pineo G, Chen D, Ramirez LM. Apixaban versus enoxaparin for thromboprophylaxis after hip replacement. $N$ Engl J Med. 2010;363(26):2487-2498. doi:10.1056/NEJ Moa1006885

23. Lassen MR, Raskob GE, Gallus A, Pineo G, Chen D, Hornick P. Apixaban versus enoxaparin for thromboprophylaxis after knee replacement (ADVANCE-2): a randomised double-blind trial. Lancet. 2010;375(9717):807-815. doi:10.1016/S0140-6736(09)62125-5

24. Lassen MR, Raskob GE, Gallus A, Pineo G, Chen D, Portman RJ. Apixaban or enoxaparin for thromboprophylaxis after knee replacement. $N$ Engl J Med. 2009;361(6):594-604. doi:10.1056/NEJMo a0810773

25. Agnelli G, Buller HR, Cohen A, et al. Apixaban for extended treatment of venous thromboembolism. N Engl J Med. 2013;368(8):699708. doi:10.1056/NEJMoa1207541 
26. Granger $\mathrm{CB}$, Alexander $\mathrm{JH}$, McMurray JJ, et al. Apixaban versus warfarin in patients with atrial fibrillation. $N$ Engl J Med. 2011;365 (11):981-992. doi:10.1056/NEJMoa1107039

27. Connolly SJ, Eikelboom J, Joyner C, et al. Apixaban in patients with atrial fibrillation. $N$ Engl J Med. 2011;364(9):806-817. doi:10.1056/ NEJMoa1007432

28. Giugliano RP, Ruff CT, Braunwald E, et al. Edoxaban versus warfarin in patients with atrial fibrillation. $N$ Engl $J$ Med. 2013;369 (22):2093-2104. doi:10.1056/NEJMoa1310907

29. Investigators E, Bauersachs R, Berkowitz SD, et al. Oral rivaroxaban for symptomatic venous thromboembolism. N Engl J Med. 2010;363 (26):2499-2510. doi:10.1056/NEJMoa1007903

30. Eikelboom JW, Connolly SJ, Bosch J, et al. Rivaroxaban with or without aspirin in stable cardiovascular disease. $N$ Engl $J$ Med. 2017;377(14):1319-1330. doi:10.1056/NEJMoa1709118

31. Fuji T, Fujita S, Kawai Y, et al. Safety and efficacy of edoxaban in patients undergoing hip fracture surgery. Thromb Res. 2014;133 (6):1016-1022. doi:10.1016/j.thromres.2014.03.009

32. Chung N, Jeon HK, Lien LM, et al. Safety of edoxaban, an oral factor Xa inhibitor, in Asian patients with non-valvular atrial fibrillation. Thromb Haemost. 2011;105(3):535-544. doi:10.1160/TH1007-0451

33. Hokusai VTEI, Buller HR, Decousus H, et al. Edoxaban versus warfarin for the treatment of symptomatic venous thromboembolism. N Engl J Med. 2013;369(15):1406-1415. doi:10.1056/NEJMoa130 6638

34. Schulman S, Kearon C, Kakkar AK, et al. Dabigatran versus warfarin in the treatment of acute venous thromboembolism. $N$ Engl J Med. 2009;361(24):2342-2352. doi:10.1056/NEJMoa0906598

35. Kakkar AK, Brenner B, Dahl OE, et al. Extended duration rivaroxaban versus short-term enoxaparin for the prevention of venous thromboembolism after total hip arthroplasty: a double-blind, randomised controlled trial. Lancet. 2008;372(9632):31-39. doi:10.1016/ S0140-6736(08)60880-6

36. Eriksson BI, Borris LC, Friedman RJ, et al. Rivaroxaban versus enoxaparin for thromboprophylaxis after hip arthroplasty. $N$ Engl $J$ Med. 2008;358(26):2765-2775. doi:10.1056/NEJMoa0800374

37. Turpie AG, Lassen MR, Davidson BL, et al. Rivaroxaban versus enoxaparin for thromboprophylaxis after total knee arthroplasty (RECORD4): a randomised trial. Lancet. 2009;373(9676):16731680. doi:10.1016/S0140-6736(09)60734-0

38. Hori M, Matsumoto M, Tanahashi N, et al. Rivaroxaban vs. warfarin in Japanese patients with atrial fibrillation - the J-ROCKET AF study. Circ J. 2012;76(9):2104-2111. doi:10.1253/circj.cj-12-0454

39. Connolly SJ, Ezekowitz MD, Yusuf S, et al. Dabigatran versus warfarin in patients with atrial fibrillation. $N$ Engl J Med. 2009;361 (12):1139-1151. doi:10.1056/NEJMoa0905561

40. Schulman S, Kearon C, Kakkar AK, et al. Extended use of dabigatran, warfarin, or placebo in venous thromboembolism. $N$ Engl $J$ Med. 2013;368(8):709-718. doi:10.1056/NEJMoa1113697

41. Patel MR, Mahaffey KW, Garg J, et al. Rivaroxaban versus warfarin in nonvalvular atrial fibrillation. $N$ Engl J Med. 2011;365(10):883891. doi:10.1056/NEJMoa1009638

42. Fuji T, Wang CJ, Fujita S, et al. Safety and efficacy of edoxaban, an oral factor Xa inhibitor, versus enoxaparin for thromboprophylaxis after total knee arthroplasty: the STARS E-3 trial. Thromb Res. 2014;134(6):1198-1204. doi:10.1016/j.thromres.2014.09.011

43. Schulman S, Kakkar AK, Goldhaber SZ, et al. Treatment of acute venous thromboembolism with dabigatran or warfarin and pooled analysis. Circulation. 2014;129(7):764-772. doi:10.1161/CIRCULAT IONAHA.113.004450

44. Fuji T, Fujita S, Kawai Y, et al. Efficacy and safety of edoxaban versus enoxaparin for the prevention of venous thromboembolism following total hip arthroplasty: STARS J-V. Thromb J. 2015;13:27. doi:10.1186/s12959-015-0057-x
45. Schulman S, Kearon C. Subcommittee on control of anticoagulation of the S, standardization committee of the international society on $\mathrm{T}$, haemostasis. definition of major bleeding in clinical investigations of antihemostatic medicinal products in non-surgical patients. J Thromb Haemost. 2005;3(4):692-694. doi:10.1111/j.1538-7836.2005.01204.x

46. Ntaios G, Papavasileiou V, Makaritsis K, Vemmos K, Michel P, Lip GYH. Real-world setting comparison of nonvitamin-K antagonist oral anticoagulants versus vitamin-K antagonists for stroke prevention in atrial fibrillation: a systematic review and meta-analysis. Stroke. 2017;48(9):2494-2503. doi:10.1161/STROKEAHA.117.017549

47. Chai-Adisaksopha C, Hillis C, Isayama T, Lim W, Iorio A, Crowther M. Mortality outcomes in patients receiving direct oral anticoagulants: a systematic review and meta-analysis of randomized controlled trials. J Thromb Haemost. 2015;13(11):2012-2020. doi:10.1111/jth.13139

48. Inohara $\mathrm{T}$, Xian $\mathrm{Y}$, Liang $\mathrm{L}$, et al. Association of intracerebral hemorrhage among patients taking non-vitamin $\mathrm{K}$ antagonist vs vita$\min \mathrm{K}$ antagonist oral anticoagulants with in-hospital mortality. JAMA. 2018;319(5):463-473. doi:10.1001/jama.2017.21917

49. Chai-Adisaksopha C, Crowther M, Isayama T, Lim W. The impact of bleeding complications in patients receiving target-specific oral anticoagulants: a systematic review and meta-analysis. Blood. 2014;124 (15):2450-2458. doi:10.1182/blood-2014-07-590323

50. Holster IL, Valkhoff VE, Kuipers EJ, Tjwa ET. New oral anticoagulants increase risk for gastrointestinal bleeding: a systematic review and meta-analysis. Gastroenterology. 2013;145(1):105-112 e115. doi:10.1053/j.gastro.2013.02.041

51. Abraham NS, Noseworthy PA, Yao X, Sangaralingham LR, Shah ND. Gastrointestinal safety of direct oral anticoagulants: a large population-based study. Gastroenterology. 2017;152(5):1014-1022 e1011. doi:10.1053/j.gastro.2016.12.018

52. Abraham NS, Singh S, Alexander GC, et al. Comparative risk of gastrointestinal bleeding with dabigatran, rivaroxaban, and warfarin: population based cohort study. BMJ. 2015;350:h1857. doi:10.1136/ bmj.h1857

53. Maura G, Blotiere PO, Bouillon K, et al. Comparison of the short-term risk of bleeding and arterial thromboembolic events in nonvalvular atrial fibrillation patients newly treated with dabigatran or rivaroxaban versus vitamin K antagonists: a French nationwide propensity-matched cohort study. Circulation. 2015;132(13):1252-1260. doi:10.1161/CIRCULA TIONAHA.115.015710

54. Lip GY, Keshishian A, Kamble S, et al. Real-world comparison of major bleeding risk among non-valvular atrial fibrillation patients initiated on apixaban, dabigatran, rivaroxaban, or warfarin. A propensity score matched analysis. Thromb Haemost. 2016;116(5):975986. doi:10.1160/TH16-05-0403

55. Noseworthy PA, Yao X, Abraham NS, Sangaralingham LR, McBane $\mathrm{RD}$, Shah ND. Direct comparison of dabigatran, rivaroxaban, and apixaban for effectiveness and safety in nonvalvular atrial fibrillation. Chest. 2016;150(6):1302-1312. doi:10.1016/j.chest.2016.07.013

56. Chan YH, Kuo CT, Yeh YH, et al. Thromboembolic, bleeding, and mortality risks of rivaroxaban and dabigatran in Asians with nonvalvular atrial fibrillation. J Am Coll Cardiol. 2016;68(13):13891401. doi:10.1016/j.jacc.2016.06.062

57. Bai Y, Deng H, Shantsila A, Lip GYH. Rivaroxaban versus dabigatran or warfarin in real-world studies of stroke prevention in atrial fibrillation: systematic review and meta-analysis. Stroke. 2017;48 (4):970-976. doi:10.1161/STROKEAHA.116.016275

58. Burr N, Lummis K, Sood R, Kane JS, Corp A, Subramanian V. Risk of gastrointestinal bleeding with direct oral anticoagulants: a systematic review and network meta-analysis. Lancet Gastroenterol Hepatol. 2017;2(2):85-93. doi:10.1016/S2468-1253(16)30162-5

59. Di Giosia P, Passacquale G, Petrarca M, Giorgini P, Marra AM, Ferro A. Gender differences in cardiovascular prophylaxis: focus on antiplatelet treatment. Pharmacol Res. 2017;119:36-47. doi:10.1016/j. phrs.2017.01.025 
60. Salzano A, Demelo-Rodriguez P, Marra AM, Proietti M. A focused review of gender differences in antithrombotic therapy. Curr Med Chem. 2017;24 (24):2576-2588. doi:10.2174/0929867323666161029223512

61. Connolly SJ, Crowther M, Eikelboom JW, et al. Full study report of andexanet alfa for Bleeding Associated with factor Xa inhibitors. $N$ Engl J Med. 2019;380(14):1326-1335. doi:10.1056/NEJMoa1814051

62. Rasmussen LH, Larsen TB, Graungaard T, Skjoth F, Lip GY. Primary and secondary prevention with new oral anticoagulant drugs for stroke prevention in atrial fibrillation: indirect comparison analysis. BMJ. 2012;345:e7097. doi:10.1136/bmj.e7097
63. Castellucci LA, Cameron C, Le Gal G, et al. Efficacy and safety outcomes of oral anticoagulants and antiplatelet drugs in the secondary prevention of venous thromboembolism: systematic review and network meta-analysis. BMJ. 2013;347:f5133. doi:10.1136/bmj.f5133

\section{Publish your work in this journal}

Clinical Epidemiology is an international, peer-reviewed, open access, online journal focusing on disease and drug epidemiology, identification of risk factors and screening procedures to develop optimal preventative initiatives and programs. Specific topics include: diagnosis, prognosis, treatment, screening, prevention, risk factor modification,

Submit your manuscript here: https://www.dovepress.com/clinical-epidemiology-journal systematic reviews, risk \& safety of medical interventions, epidemiology \& biostatistical methods, and evaluation of guidelines, translational medicine, health policies \& economic evaluations. The manuscript management system is completely online and includes a very quick and fair peer-review system, which is all easy to use. 